\section{Military Technical College Kobry El-Kobbah, Cairo, Egypt}

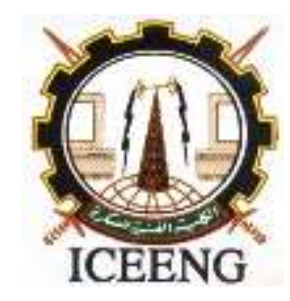

\author{
$11^{\text {th }}$ International Conference \\ on Electrical Engineering \\ ICEENG 2018
}

\title{
Tissue-Equivalent Phantoms Recognition Employing Hyperspectral Imaging
}

\author{
Ramy Abdlaty* and Shirley Deng **
}

\begin{abstract}
The incessant innovations, of hyperspectral imaging (HSI), and data mining algorithms, express the necessity for developing reliable assessment and comparison means. In medical applications of $\mathrm{HSI}$, for instance, one of the means of assessment is tissueequivalent phantoms. These phantoms are designed to mimic the spectral behavior of the real living tissues. In this work, gel-based-phantoms are prepared with altered ingredients. The gel phantom's ingredients include India ink, and Intralipid to provide absorption and scattering respectively. Unlike visual assessment, and photography, $\mathrm{HSI}$ for succeeded to identify the various phantoms based on its spectral signature. In conclusion, we introduce a simple method to evaluate the performance of newly developed optical imaging techniques including HSI via an affordable, inexpensive, and easy to make phantoms.
\end{abstract}

\section{1- Introduction}

Until then, skin diseases diagnosis and treatment evaluation are inspected by visual assessment, the gold standard [1], [2], via a dermatologist [3], [4]. However, visual assessment was criticized in many studies to be subjective, qualitative, temporally inconsistent, and invasive [5]-[10]. To overcome the critiques of visual assessment, objective techniques were proposed for precise skin inspection/ treatment assessment. The proposed objective techniques, in literature, include optical [11], and non-optical techniques [12]. In optical techniques, there are two main approaches for skin assessment; one of which is based on diffuse reflectance spectroscopic (DRS) measurements while the other is based on color imaging. The spectroscopic-basedapproach is well-known for detecting the spectral signature of the skin's symptoms by high precision, inexpensive equipment [4]. Hence, it aids the dermatologist in differentiating between visibly alike skin's symptoms. Unfortunately, spectroscopic measurements techniques need direct skin contact; which is hard to achieve in cases including burns, and limited to a small area of inspection; therefore, it takes lengthy time, and effort to judge a sizeable region of interest (ROI). Color imaging/ photography, unlike spectroscopic measurements, is contactless and deals with a sizable ROI. Nonetheless,

* Egyptian Armed Forces.

${ }^{* *}$ Faculty of Health sciences, McMaster University, Ontario, Canada. 
photography suffers from poor spectral resolution; due to its limitation of three bands: red, green and blue. Far from spectral shortage, photography is a cumbersome process since it requires firm conditions in order to achieve the expected efficiency such as illumination consistency. Taken together, the limitations of the famous optical techniques for skin assessment provoked the inspiration of spectral imaging.

Spectral imaging (SI) is a hybrid technique combining both the advantages of DRS and photography and thus, it overcomes the limitations of each [13]. HSI is used for acquiring multiple frames for an object of interest, likewise photography, however, each frame is captured at distinct wavelength [14]. As thus, the object's ROI total spectral radiance is acquired in a 3-dimensional data format, two of which are spatial and the third is spectral one. SI data analysis/ classification enables a precise estimation of the componential analysis for object under test [15]. Several considerations were taken in order to categorize SI such as the number of bands, the resolution, and the acquisition schemes. Regarding the number of bands, for example, SI is one of three: multispectral imaging (MSI, usually uses less than 10 bands) [16]-[18], hyperspectral imaging (HSI, between 10 and 1000 bands) [13], [19], [20], or ultraspectral imaging (USI, 1000 or more bands) [21]. In terms of the data acquisition, tunable filter-based-SI is common scheme for the spectral scanning scheme. Two filters are most commonly used in HSI: acousto-optic tunable filter (AOTF), and liquid crystal tunable filter (LCTF). AOTFs are distinguished by higher spectral resolution, while LCTFs are recommended for achieving high quality images. The use of tunable filters, in visible and near infrared spectra, entails the use of broadband light sources such as tungsten-halogen or xenon light sources. Chargecoupled device (CCD)/ complementary metal oxide semiconductor (CMOS) are the challenging technologies for image detection in HSI. In terms of applications, HSI has a successful role in several research fields including medicine [22]. Nevertheless, HSI system is not handy for purchasing. Therefore, custom-made HSI systems is low-cost to build but requires more effort to be validated and tested. To give an example, phantoms with tissue like properties, provided that being longstanding, and easy to reproduce, are fruitful way of optical innovative techniques assessment.

Tissue-equivalent phantoms (TEP) captivated the attention of research studies; since it is convenient in simulating real tissues [23]-[27]. Typically, TEP fabrication is dependent on wax, resin, and agarose as the base materials combined with either; milk, blood, Intralipid, or yeast suspension. To mimic the natural absorbers/ diffusers in biologic tissues, ink and polystyrene particles are added to the mixture [23], [25], [27]-[29]. TEP can be customized to mimic the spectral signature of certain tissue in a minute band such as red $(633 \mathrm{~nm})$ [30], [31] or a wider portion of the spectrum like near-infrared [32]. In this work, we test a custom-made AOTF-HSI platform, originally designed by our group for skin erythema assessment, by using visually alike, but physically different TEP. The test results aid in evaluating the effectiveness of the developed AOTF-HSI system for a real assessment regarding radiotherapy induced erythema in skin cancer treatment.

\section{2- Materials and Methods}

2.1- Hyperspectral Imaging Instrument 
Conveniently, HSI setup uses a wide-band light source, to illuminate the object of interest. The light source, used here, is a set of two commercial tungsten-halogen lamps with total power of 300 watt. The reflected photons back from the illuminated ROI, within the field of view (FOV) of the instrument, are collected and directed toward an optical filter positioned ahead of an image detector to scan through the successive spectral bands of operation. To give more details, Figure 1 displays the schematic diagram for the developed AOTF-HSI optical setup in the current work. In the lead, there is a zoom lens (Cannon EF-S 55-250 mm f/4-5.6 IS) installed in order to collect the reflected photons. These photons, within the lens FOV, are focused to form the object image in its focal plane. The aforesaid plane is adjusted to be at the focal plane for the second lens in the HSI optical setup. The second lens is the leading lens of an optical relay, formed of two typical achromatic lenses, acting as image transporter from the first focal plane to the detector screen. The optical relay lenses are achromatic in order to avoid the chromatic aberration. In between the relay lenses, a polarizing beam splitter (PBS) is installed. The PBS function is to modify the randomly polarized input beam of photons into two orthogonally polarized rays. One ray, of the two orthogonal rays, is transmitted through the PBS while the second is perpendicularly reflected.

For high throughput purpose, a mirror followed by a half-wave plate $(\lambda / 2)$ are installed on the reflecting side of the PBS. The reason for installing the mirror and the half-wave plate is to modify the reflected ray in both direction and polarization. By this modification, the two rays of the input beam of photons become not only polarized but also matching the the AOTF polarization, and injected within its acceptance angle. The incorporated AOTF (Gooch \& Housego TF625-350-2-11-BR1A), here, is a noncollinear configuration. The AOTF crystal is made of tellurium oxide crystal $\left(\mathrm{TeO}_{2}\right)$ with an input aperture of $11 \times 12$ $\mathrm{mm}$ window. The filter is operating in the spectral range of $450-800 \mathrm{~nm}$. An 8-channel digital frequency synthesizer (Gooch \& Housego MSD040-150-0.2ADM-A5H-8 x 1) drives the AOTF device with a radio frequency (RF) signal ranges from $40-150 \mathrm{MHz}$ The RF driver is capable of simultaneously emitting 8 separate frequencies through the $\mathrm{TeO}_{2}$ crystal, by which 8 spectral bands are diffracted. A set of control commands are used to adjust the tuning RF signal. These commands are sent through a universal serial bus protocol via a work station. This work station controls not only the AOTF device, but also the image detector. The image detector (Ximea MQ042rg-CM Enhanced-IR), employed in the optical setup, is a monochromatic complementary metal oxide semiconductor (CMOS) camera, with a sensor size of $11.24 \mathrm{~mm}^{2}$. The whole components are well fixed to a metal platform to ensure the stability and rigidity of the HSI system during both movement and operation. 


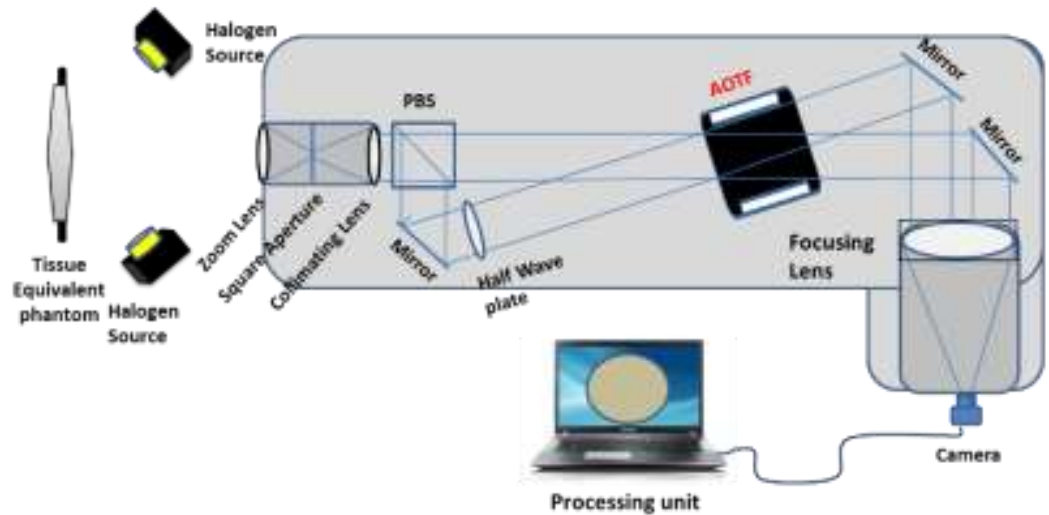

Figure 1: A schematic diagram displays hyperspectral imaging (HSI) system configuration used for AOTF and LCTF comparison. The optical components used to build this setup are: zoom lens, square aperture, two achromatic (collimating and focusing) lenses, flat mirrors; PBS: polarizing beam splitter; half-wave plate, and a camera for capturing images, and computer for system control and data storage. All the former components are tightly fixed to a metal platform to ensure the setup's stability and portability.

\section{2-Tissue-equivalent phantom preparation}

For reliability and marketing purposes, the newly developed optical instrumentation including $\mathrm{HSI}$ need to be tested and characterized prior operation. One sort of instrumentation testing is via the use of TEP's. These phantoms are characterized by a typical range of tissue's optical properties. The easiest way of developing phantoms are the aqueous suspensions, which are typically composed of Intralipid and India ink [24]. Although liquid phantoms are intensely adaptable, it is hard to include heterogeneities. Therefore, several hydrogel-based phantoms mediated by agar, or gelatin offered the opportunity to form distinct shapes and formats of phantoms.

Gel-based-phantoms, here, were constructed with identified concentrations of an absorber (water soluble India ink) and scatterer (intralipid). The gel format of the phantoms is controlled by using agar (R9012HR, Cedarlane Laboratories, Burlington, ON, $\mathrm{CA}$ ) solution by diluting agarose powder with a weight ratio of $1.0 \%$. To verify the efficacy of India-ink contributions used to prepare the phantom construction, samples of altered ink solutions were prepared, displayed in Figure 2-(a), and characterized by two ways.

The first way is using a custom-made setup, shown in Figure 2-(b), composed of a broad band light source, pinhole, objective lens, sample, and a fiber based spectrophotometer (NEWPORT Model OSM400, Irvine, Ca, USA). The setup used a broad band light source to illuminate the sample via a pinhole to clear the undesired optical noise. An objective lens is placed at one focal length from the pinhole to to collimate the beam of light toward the sample. The collimated beam, used to illuminate the ink samples is shaped using a square aperture. The transmitted light is detected and measured via an optical fiber, $(\phi$ $400 \mu \mathrm{m}$, and $0.22 \mathrm{NA}$, Thorlabs, Newton, NJ, USA) connected to a computer controlled 
spectrophotometer. The second way of verification is using a high standard calibrated spectrometer (GENYSIS ${ }^{\text {TM }}$ 10S UV-Vis, Thermo Fisher Scientific, Waltham, Ma, USA). Dilutions of the absorber component were done using a stock solution of water soluble India ink as shown in Figure 2. Toward constructing distinct phantoms, the level of optical absorption value $\left(\mathrm{mm}^{-1}\right)$ is changed in each phantom by increasing the ink incrementally while holding the reduced scattering at a constant value.

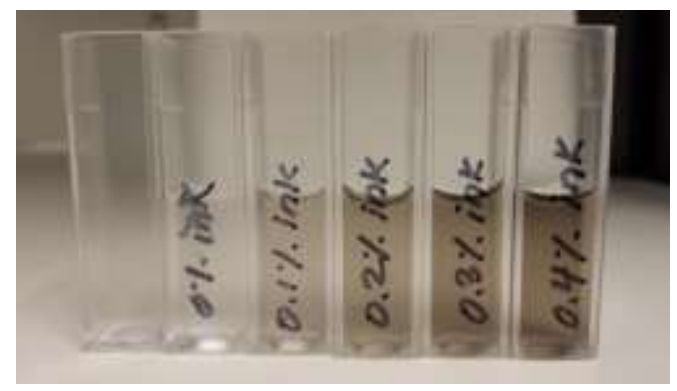

(a)

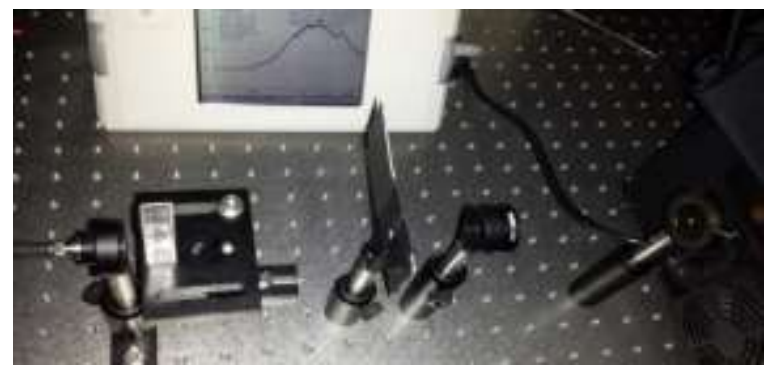

(b)

Figure 2: (a) Dilutions of the India ink component, used in developing the gel-based phantoms, were done using a stock solution of water soluble India ink. (b) A custom-made optical setup for measuring the absorbance of varying solution of water soluble India ink.

In preparation process of TEP, the agar powder $(0.2 \mathrm{gram})$ is added to $9.8 \mathrm{~mL}$ of distilled water, and then heated in a water path $\left(85^{\circ} \mathrm{C}\right)$ for 50 minutes. In parallel, $8.6 \mathrm{~mL}$ of water, $1.2 \mathrm{~mL}$ of $20 \%$ stock Intralipid solution, and $0.2 \mathrm{~mL}$ of India ink are mixed and poured into a $10 \mathrm{~mL}$ test tube. Once the agarose solution becomes transparent, the mixture containing the Intralipid is also put in the water path for 10 minutes. After then, the two, $10 \mathrm{~mL}$ test tubes are combined in a $50 \mathrm{~mL}$ test tube to be well mixed. At this point, fast work is critical because the gel will rapidly solidify once it is removed from the heated water path. Finally, pour the $20 \mathrm{~mL}$ mixed solution into the $20 \mathrm{~mL}$ phantom to form the shape shown in Figure 3. The whole process of phantom construction was carefully considered in each stage to achieve the required reliability. 




Figure 3: Tissue equivalent phantom (TEP's) prepared with same ingredients (Agarose, Intralipid, India ink, and distilled water) except the India ink concentrations. The ink concentrations percentages are from left to right and from down to up are $(0.0,0.1,0.2$, $0.30 .4,0.5 \%$ ) respectively.

\section{3- Optical measurements}

The optical measurement implemented, here, commenced with verifying the accuracy of preparing samples of water soluble India ink. Five samples $(0 \%, 0.1 \%, 0.2 \%, 0.3 \%$, and $0.4 \%$ ) were used in the verification process. The measured light intensities before and after inserting the samples were recorded. Based on Beer-Lambert's law, displayed in Eq. (1), absorption was computed and plotted. Based on the former measurement, TEP, with altered India ink concentration, were prepared. Afterwards, TEP were illuminated by a wide band light source and imaged by the developed HSI device. An entire datacube were recorded for each TEP. Instantaneously, each image cycle for a set of TEP's, was followed by capturing two datacubes. The first cube of images is taken for a white standard reflectance target (Labsphere-SRS-99), while the second datacube is for the sensor dark noise. The latter cube is recorded while the camera shutter and all the room lights are turned off. The reflectance.

$$
A=-\log \frac{I}{I_{o}}
$$

\section{3- Experiments and Results}

The prepared samples of ink solutions were characterized in terms of light transmittance through samples. The results of the computed light intensity demonstrated an agreement with the amounts of the India ink added to each sample. as shown in Figure 4. Based on the attained results for sole India ink sole soluble solutions, the TEP's were prepared in the lab for multiple times. Other than India ink, the agarose and intralipid ratios were adjusted to achieve the humidity and the stability of the phantoms. The stability of the phantoms is of great importance in the imaging process since the employed HSI device is, unlike microscope, horizontally imaging. The captured data is stored in the form of data sets/ cubes for each phantom combined with the dark and the white standard target cubes. 


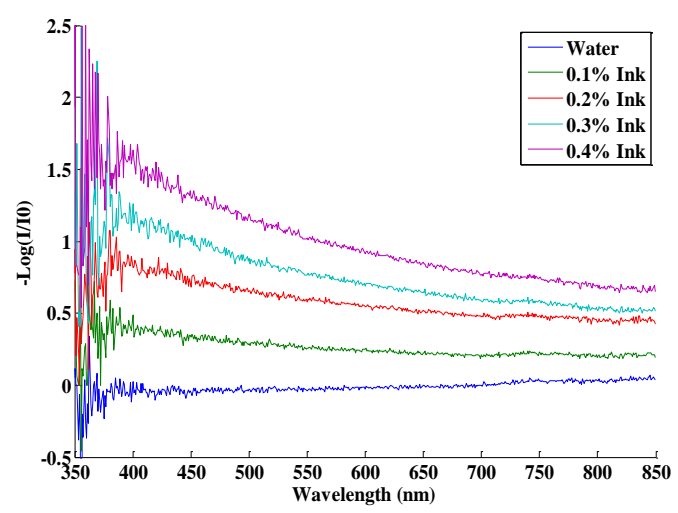

(a)

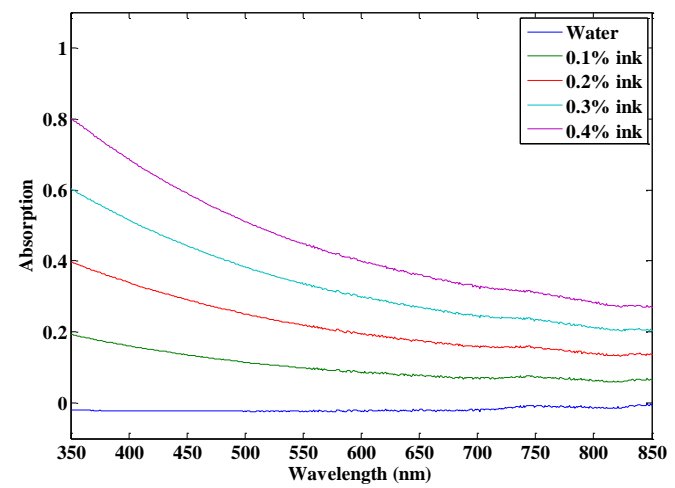

(b)

Figure 4: the computed light absorption based on beer-Lambert's law for the prepared India ink soluble water solutions by (a) custom-made spectrometric measurement, and (b) high standard calibrated spectrometer (GENYSISTM 10S UV-Vis, Thermo Fisher Scientific, Waltham, Ma, USA).

The TEP's datacubes were processed: first, by detector noise removal via subtracting the dark cubes. Afterwards, the TEP's datacubes are normalized to the formerly mentioned white standard reflectance target datacube, in order to accommodate for uneven illumination effects and the spectral dependence of the imaging setup applying Eq. (2). Where $R_{n}$ is the normalized reflectance, $R_{s 99}$ is the measured back reflected intensity of the white standard, and $R_{T E P}$ is the reflectance of the TEP. The value for $R_{n}$ is plotted against one wavelength $(\sim 700 \mathrm{~nm})$ to show how the reflectance of the prepared phantoms are interrelated together as shown in Figure 5.

$$
R_{n}=\frac{R_{T E P}-R_{D}}{R_{S 99}-R_{D}}
$$




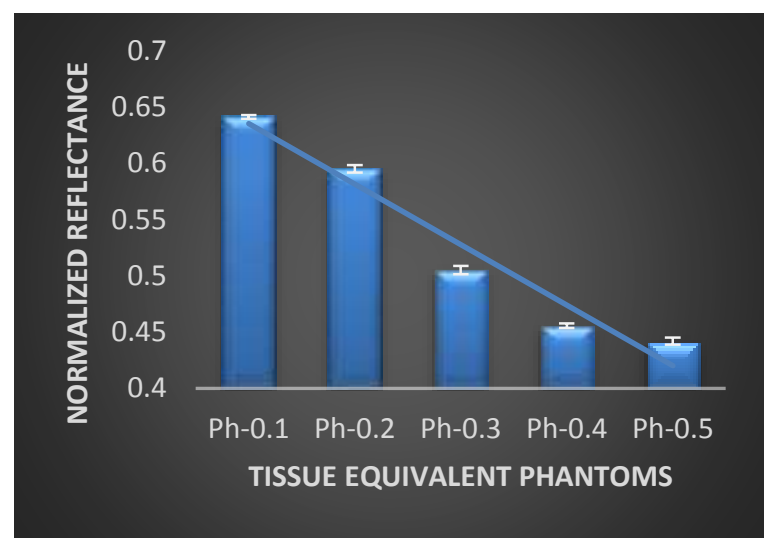

Figure 5: Computed TEP's reflectance at the spectral band of the centered $696 \mathrm{~nm}$ wavelength for 5 phantoms with gradual increase in India ink ingredients. A linear trendline is drawn to display the error between the computed reflectance and the ideal one.

\section{4- Discussion}

Past, current, and continued efforts are exerted to compose phantoms in order to mimic human tissues' behavior. These efforts are recorded in many studies in literature [33]. Literally, the aqueous solution of a scattering medium, including Intralipid, and a watersoluble India ink is designated as one of the top popular sort of biological simulating phantoms. However, this sort of phantoms suffers from the disadvantage of the incapability of creating layered structures. To overcome the previously mentioned disadvantage, agarose component is involved in synthesizing phantoms. The agar component allowed the development of multilayered structures where absorbing and scattering ingredients are included. Likewise, the phantoms prepared for this work can be molded into any custom shape.

The work, implemented here, made use of the agar-based phantoms to assess the sensitivity of $\mathrm{HSI}$ in detecting changes in embeded chromophores concentration within the visible and the near infrared region $(450: 800 \mathrm{~nm})$. The variation of the ink dye embeded in the phantoms, used here, mimics the process of the human skin optical properties change due to the variation of its encompassed chromophores. To verify the reliability of the optical properties of the phantoms, the preparation process was repeated and revised provided that the absorbing and scattering components are accurately speckled. The revised steps of preparation include checking the macroscopic homogeneity of the phantom ingredients by measuring the transmittance as has been done with the India ink in Figure 4.

The procedure we introduce, in this work, facilitates the development of a phantom which is straightforward in manufacturing, usage, and molding in alternate formats. The former advantages of the phantom make it useful in biomedical studies, for example, offering the required in vivo environment, likewise contrast the light-transport models with empirical measurements. To give this work more sense, the phantoms, used here, proved the feasibility of employing $\mathrm{HSI}$ in a research study, currently in its way at McMaster university, in order to diagnose the skin lesions treated via radiotherapy. Figure 5 
illustrates the capability of $\mathrm{HSI}$ for differentiating between various TEP while visual assessment is incapable.

\section{5- Conclusion}

In a nutshell, the study presented here in this paper tagged the AOTF-HSI, with the capability of differentiating between TEP's with dissimilar ingredients. As a consequence, the decision of using HSI becomes more feasible in skin diseases diagnosis. The major advantages of AOTF is summarized in the following points:

AOTF-HSI configuration is distinguished by:

a. Fine spectral resolution

b. Significant out of band suppression

c. Fast wavelength access, appropriate for video rate

d. High diffraction efficiency $(90 \%)$

e. Compact size

f. Simultaneous diffraction of 8-distinct wavelengths

Ultimately, we need to emphasize that this work is a preliminary step toward skin erythema objective assessment. In general, HSI system robust design is application based. For medical applications, as an example, if the biological tissue of interest has unknown optical properties, or characterized by close spectral features, AOTF-HSI might be the recommended option.

\section{Aknowledgement}

The ingredients of the tissue equivalent phantoms used in the experiments were kindly provided by Professor Qiyin Fang and described by Sharon Goh.

\section{References}

[1] A. S. C. J. Taylor S.C., "The taylor hyperpigmentation scale: A new visual assessment tool for the evaluation of skin color and pigmentation," Ther. Clin., vol. 76, no. 4, pp. 270-274, 2005.

[2] D. Basketter, F. Reynolds, M. Rowson, C. Talbot, and E. Whittle, "Visual assessment of human skin irritation: a sensitive and reproducible tool.," Contact Dermatitis, vol. 37, pp. 218-220, 1997.

[3] S. G. Kong, M. Martin, and T. Vo-Dinh, "Hyperspectral Fluorescence Imaging for Mouse Skin Tumor Detection," ETRI J., vol. 28, no. 6, pp. 770-776, Dec. 2006.

[4] D. L. Glennie, J. E. Hayward, D. E. Mckee, and T. J. Farrell, "Inexpensive diffuse reflectance spectroscopy system for measuring changes in tissue optical properties," J. Biomed. Opt., vol. 19, no. December, pp. 105005-1-105005-6, 2014.

[5] A. D. Ormerod, C. M. Dwyer, R. Weller, D. H. Cox, and R. Price, "A comparison of subjective and objective measures of reduction of psoriasis with the use of ultrasound, reflectance colorimetry, computerized video image analysis, and nitric oxide production," J. Am. Acad. Dermatol., vol. 37, no. 1, pp. 51-57, 1997.

[6] J. Nyström, P. Geladi, B. Lindholm-Sethson, J. Rattfelt, A. C. Svensk, and L. Franzen, "Objective measurements of radiotherapy-induced erythema," Ski. Res. 
Technol., vol. 10, no. 4, pp. 242-250, 2004.

[7] G. V Oliveira, D. Chinkes, C. Mitchell, G. Oliveras, H. K. Hawkins, and D. N. Herndon, "Objective assessment of burn scar vascularity, erythema, pliability, thickness, and planimetry.," Dermatol. Surg., vol. 31, pp. 48-58, 2005.

[8] A. Raina, R. Hennessy, M. Rains, J. Allred, D. Diven, and M. K. Markey, "Objective measurement of erythema in psoriasis using digital color photography with color calibration," Ski. Res. Technol., no. 7, pp. 1-6, 2015.

[9] L. Diana L. Glennie, Joseph E. Hayward, Orest Z. Ostapiak, James Wright and T. J. F. Doerwald-Munoz, "Diffuse reflectance spectroscopy for monitoring erythema in head \& neck intensity modulated radiation therapy," J. Radiat. Oncol., 2014.

[10] A. Madooei et al., "Hyperspectral Image Processing for Detection and Grading of Skin Erythema," in SPIE_MI.

[11] N. Kollias and G. N. Stamatas, "Optical non-invasive approaches to diagnosis of skin diseases," J. Investig. Dermatology Symp. Proc., vol. 7, pp. 64-75, 2002.

[12] B. Jung, S. Kim, Y. Bae, H. Kang, Y. Lee, and J. S. Nelson, "Real-time measurement of skin erythema variation by negative compression: pilot study.," J. Biomed. Opt., vol. 17, no. 8, pp. 81422-1, Aug. 2012.

[13] K. B. Yushkov and V. Y. Molchanov, "Hyperspectral imaging acousto-optic system with spatial filtering for optical phase visualization," J. Biomed. Opt., vol. 22, no. 6, p. 66017, 2017.

[14] R. E. Warren and D. B. Cohn, "Chemical detection on surfaces by hyperspectral imaging," J. Appl. Remote Sens., vol. 11, no. 1, p. 15013, 2017.

[15] G. Lu, D. Wang, and Z. G. Chen, "Spectral-spatial classification for noninvasive cancer detection using hyperspectral imaging detection using hyperspectral imaging," J. Biomed. Opt., vol. 19(10), 2014.

[16] L. Kong et al., "Handheld erythema and bruise detector," Proc. SPIE, vol. 6915, p. 69153K-69153K-7, 2008.

[17] D. Jakovels, J. Spigulis, and I. Saknite, "Multi-spectral mapping of in vivo skin hemoglobin and melanin," SPIE Proc., vol. 7715, p. 77152Z--77152Z, 2010.

[18] L. Kong et al., "Single sensor that outputs narrowband multispectral images.," J. Biomed. Opt., vol. 15, no. 1, p. 10502, 2010.

[19] S. Miclos, S. V. Parasca, M. A. Calin, D. Savastru, and D. Manea, "Algorithm for mapping cutaneous tissue oxygen concentration using hyperspectral imaging," Biomed. Opt. Express, vol. 6, no. 9, pp. 3420-3430, 2015.

[20] B. S. Sorg, B. J. Moeller, O. Donovan, Y. Cao, and M. W. Dewhirst, "Hyperspectral imaging of hemoglobin saturation in tumor microvasculature and tumor hypoxia development.," J. Biomed. Opt., vol. 10, no. 4, p. 44004, 2014.

[21] a. D. Meigs, I. Otten, L.J., and T. Y. Cherezova, "Ultraspectral imaging: a new contribution to global virtual presence," in 1998 IEEE Aerospace Conference Proceedings (Cat. No.98TH8339), 1998, vol. 2, no. October.

[22] G. Lu and B. Fei, "Medical hyperspectral imaging: a review.," J. Biomed. Opt., vol. 19, no. 1, p. 10901, Jan. 2014.

[23] J. C. Ramirez-san-juan, B. Choi, W. Franco, J. S. Nelson, and G. Aguilar, "Effect of ambient humidity on light transmittance through skin phantoms during cryogen spray cooling," Phys. Med. Biol., vol. 50, pp. 1-8, 2005.

[24] J. Pekar and M. S. Patterson, "Fabrication and characterization of phantoms with 
tissue-like optical properties from 500 to 700 nm," Med. Laser Appl., vol. 25, pp. 147-153, 2010.

[25] N. Chahat, M. Zhadobov, S. Alekseev, and R. Sauleau, "Human skin-equivalent phantom for on-body antenna measurements in $60 \mathrm{GHz}$ band," Electron. Lett., vol. 48, no. 2, pp. 9-10, 2012.

[26] J. R. Weber et al., "Multispectral imaging of tissue absorption and scattering using spatial frequency domain imaging and a computed-tomography imaging spectrometer.," J. Biomed. Opt., vol. 16, no. 1, p. 11015, 2011.

[27] I. Nishidate, T. Maeda, K. Niizeki, and Y. Aizu, "Estimation of melanin and hemoglobin using spectral reflectance images reconstructed from a digital RGB image by the Wiener estimation method," Sensors (Switzerland), vol. 13, no. 6, pp. 7902-7915, 2013.

[28] J. W. Pickering, S. a Prahl, N. van Wieringen, J. F. Beek, H. J. Sterenborg, and M. J. van Gemert, "Double-integrating-sphere system for measuring the optical properties of tissue.," Appl. Opt., vol. 32, no. 4, pp. 399-410, 1993.

[29] S. C. Gebhart, R. C. Thompson, and A. Mahadevan-Jansen, "Liquid-crystal tunable filter spectral imaging for brain tumor demarcation," Appl. Opt., vol. 46, no. 10, p. 1896, 2007.

[30] M. S. Patterson, B. Chance, and B. C. Wilson, "Time resolved reflectance and transmittance for the non-invasive measurement of tissue optical properties," Appl. Opt., vol. 28, no. 12, pp. 2331-2336, 1989.

[31] W. M. S. M. J. C. VAN GEMERT, STEVEN L. JACQUES, H. J. C. M. STERENBORG, "Skin Optics," IEEE Trans. Biomed. Eng., vol. 36, no. 12, pp. 1146-1154, 1989.

[32] M. Firbankt, M. Odq, and D. T. Delpyt, "An improved design for a stable and reproducible phantom material for use in near-infrared spectroscopy and imaging," Physc. Med. Biol., vol. 40, pp. 955-961, 1995.

[33] M. Lualdi, A. Colombo, B. Farina, and S. Tomatis, "A Phantom With Tissue-Like Optical Properties in the Visible and Near Infrared for Use in Photomedicine," Lasers Surg. Med., vol. 28, no. July 2000, pp. 237-243, 2001. 